\title{
Prognostic Value of Gastrokine-2 (GKN2) and Its Correlation with Tumor-Infiltrating Immune Cells in Lung Cancer and Gastric Cancers
}

This article was published in the following Dove Press journal: Journal of Inflammation Research

\author{
Fangteng Liu ${ }^{1,2}$ \\ Hengyu Wu' \\ 'Department of Breast Surgery, The \\ Third Hospital of Nanchang, Nanchang, \\ Jiangxi 330009, People's Republic of \\ China; ${ }^{2}$ Faculty of Medicine, University of \\ Munich, Munich 80336, Germany
}

Background: GKN2, as a secretory protein, is involved in the inflammation and immune modulation, and its aberrant expression is closely related to tumorigenesis. However, integrated studies on the value of GKN2 as a promising clinical biomarker and immunotherapy target in multiple tumors are still rare.

Methodology: Multiple online databases, including ONCOMINE, SEGreg, UALCAN, GEPIA, K-M Plotter, cBioPortal, MethSurv, CellMarker, and Timer, were applied to assess the clinical significance of GKN2 and its correlation with tumor-infiltrating immune cells in differentially expressed cancers.

Results: Several databases confirmed that GKN2 was significantly down-regulated in lung and gastric cancers compared that in normal samples. GKN2 was altered in 3\%, 5\%, and 4\% of the LUAD, LUSC, and STAD samples, respectively. Hyper-methylation of GKN2 was found in LUAD and LUSC samples. For the clinical values of GKN2, we found that the low transcription level of GKN2 was associated with worse OS in lung cancer, and inferior FP and PPS in gastric cancer, and the relationships between GKN2 expression and clinical variables regarding OS/FP/PPS in lung and gastric cancers were assessed. Moreover, the prognostic value of the DNA methylation patterns of GKN2 in LUAD, LUSC, and STAD was identified. Furthermore, GKN2 expression was found to be significantly correlated with the infiltrating multiple tumor immune cells, and statistically significant differences in the correlation between GKN2 expression and multiple markers of neutrophils and macrophage polarization were observed in LUAD, LUSC, and STAD.

Conclusion: The study revealed the prognosis and risk factors for deterioration in patients with low expression of GKN2. GKN2 may be used as a valuable prognostic biomarker and therapeutic target in lung and gastric cancers.

Keywords: GKN2, lung cancer, gastric cancer, prognosis, tumor-infiltrating, biomarker

\section{Introduction}

Gastrokine 2 (GKN2), also previously called GDDR, is a protein-encoding gene mapped to chromosome 2 p13 with five exons. It was firstly cloned and reported by Du et al in 2003. ${ }^{1}$ GKN2 encodes an 18.3-kDa secreted protein, it contains an approximately 100 amino acid brichos domain and homology with smart00019.10, SF_P. ${ }^{2}$ GKN2 protein belongs to the gastrokine family proteins that share $60 \%$ similar protein sequences. ${ }^{3}$ GKN2 was originally found to be abundant in normal gastric mucosa but downregulated or absent in gastric cancer tissue. ${ }^{1}$ Moss et al also found that GKN2 expression was frequently lost in gastric cancer, especially in
Correspondence: Hengyu Wu

Tel +86- I5270280590

Email why_wuhengyu0590@163.com
Journal of Inflammation Research 2020:13 933-944 
the diffuse subtype. ${ }^{4}$ GKN2 low expression was also observed in other malignant tumors, such as lung cancer, colon cancer, and glioblastoma, ${ }^{5-7}$ but was found upregulated in breast and esophageal cancer compared with that in normal tissues. ${ }^{8-11}$ These showed the different expression patterns of GKN2 across human various types of tumors.

Multiple studies reported that GKN2 was associated with clinical outcomes, and represent a therapeutic target for human cancers. Low expression of GKN2 mRNA was found to be associated with inferior relapse-free survival in lung adenocarcinoma, ${ }^{12}$ and worse disease-free survival in breast cancer, ${ }^{13}$ shorter disease-specific survival in colorectal cancer. ${ }^{6}$ GKN2 loss promoted gastric tumorigenesis and cancer metastasis, and otherwise, GKN2 overexpression suppressed the proliferation, migration, and invasion of gastric cancer cells. ${ }^{14}$ Besides, low expression of GKN2 was associated with poor overall survival in gastric cancer. ${ }^{14}$ These findings suggested that GKN2 has important impacts on the initiation of cancer and development.

GKN2 contributes to the maintaining of homeostasis and is involved in the immune response. ${ }^{15,16}$ GKN2 was found to be a glucocorticoid-responsive enhancer element in glucocorticoid signaling, coordinate expression loss of GKN1 and GKN2 in gastric cancer via impairment of a glucocorticoid-responsive enhancer. ${ }^{17}$ And GKN2 could recruit neutrophils and promote the release of inflammatory factors to contribute to inflammation, GKN2 attenuates acute gastric lesions through the NLRP3 Inflammasome. ${ }^{18}$ GKN2 may contribute to the homeostasis of gastric epithelial cells by inhibiting GKN1 activity. ${ }^{19}$ These suggested that GKN2 has an effect on the regulation of immune infiltrating cells and is involved in the tumor microenvironment. Nevertheless, the potential exact roles of GKN2 in tumorigenesis and microenvironment are undefined.

In this work, we comprehensively compared the expression level in various kinds of tumors vs normal tissues and determined the differentially expressed cancers. And then explored the prognostic values of the transcription level and DNA methylation of GKN2 in these cancers, the relationship between GKN2 expression and clinical variables was also assessed. At last, we explored the correlation between GKN2 and tumor-infiltrating immune cells to elucidate the potential mechanism of GKN2 in tumor progression and prognosis. The flow diagram was presented in Supplementary Figure 1.

\section{Materials and Methods GEPIA}

GEPIA (http://gepia.cancer-pku.cn/index.html) ${ }^{20}$ was applied for interactive body map, the gene expression profile of GKN2, pathological stage analysis of GKN2, and correlation analysis between GKN2 and immune marker genes in lung adenocarcinoma (LUAD), lung squamous cell carcinoma (LUSC), and stomach adenocarcinoma (STAD).

\section{SEGreg}

SEGreg (http://bioinfo.life.hust.edu.cn/SEGreg) is a comprehensive database to identify the specifically expressed genes in cancers/tissues/cell lines (TCGA, BodyMap, CCLE, and GTEx) from several databases. ${ }^{21}$

\section{ONCOMINE}

ONCOMINE (www.oncomine.org) database was used to evaluate the expression of GKN2 in various cancers according to the significance thresholds ( $\mathrm{p}$-value 0.05 , fold change1.5, gene rank the top $10 \%)^{22}$

\section{UALCAN}

In this work, UALCAN (http://ualcan.path.uab.edu/analy sis.html) database was applied to investigate the expression of GKN2 and methylation level of GKN2 in lung cancer and gastric cancer. ${ }^{23}$

\section{cBioPortal}

cBioPortal (www.cbioportal.org) was used to explore the genetic alterations of GKN2 in LUAD, LUSC, and STAD. ${ }^{24,25}$

\section{Kaplan-Meier Plotter}

In this work, Kaplan-Meier Plotter (https://kmplot.com/ analysis/) was used to explore the clinical values of GKN2 in patients with lung and gastric cancer. ${ }^{26}$ OS (overall survival), FP (first progression), and PPS (postprogression survival) and multiple clinical characteristics were assessed in lung and gastric cancer.

\section{MethSurv}

MethSurv (https://biit.cs.ut.ee/methsurv/) web tool ${ }^{27}$ was applied to perform multivariable survival analysis using DNA methylation data of GKN2 in LUAD, LUSC, and STAD using TCGA. 


\section{Timer}

Timer web server (https://cistrome.shinyapps.io/timer/) ${ }^{28,29}$ was used to explore the correlation of GKN2 level and the infiltration of the immune cells with the "Gene module", the cox proportional hazard model was also conducted. The correlations between somatic copy number alterations of GKN2 and abundance of immune infiltrates were analyzed under "SCNA module", the correlation module was used to draw the expression scatterplots between GKN2 and genes of macrophage polarization markers in LUAD, LUSC, and STAD, the related gene markers of monocytes and macrophage were obtained from related references ${ }^{30-33}$ and the CellMarker database (http://biocc.hrbmu.edu.cn/ CellMarker/). ${ }^{34}$

\section{Statistical Methods}

Kaplan-Meier plots were used to get curves related to overall survival (OS), first progression (FP), and postprogression survival (PPS). The results from Oncomine were displayed with the number of analyses, gene rankings, fold changes, and P-values. The data were from KaplanMeier plotter, MethSurv, GEPIA, and TIMER were shown with the log-rank P-value with the hazard ratio (HR). Gene correlations were evaluated with Spearman correlation coefficients and P-values. A P-value of less than 0.05 was considered statistically significant.

\section{Results \\ GKN2 Expression Level in Human Tumors and Normal Tissues}

We explored the gene expression distribution of GKN2 across all tumor samples and paired normal tissues. The interactive body-map was visually shown the median expression of GKN2 in tumor and normal samples (Figure 1A). And the transcription levels of GKN2 were noticeably higher in STAD, and followed by LUAD and LUSC among all cancers (Figure 1B). In all normal tissues, the gene expression level of GKN2 was remarkedly higher in the stomach and followed by lung tissue (Figure 1C).

And then we compared the GKN2 expression level in human tumors vs corresponding normal tissues. In the Oncomine database, significantly more datasets showed low expression of GKN2 in tumor versus normal samples than the high expression of GKN2 (Figure 2A). The absolutely low expression of GKN2 was observed in eight kinds of malignant tumors, including brain and CNS cancer, colorectal cancer, gastric cancer, lung cancer, myeloma, and sarcoma, while
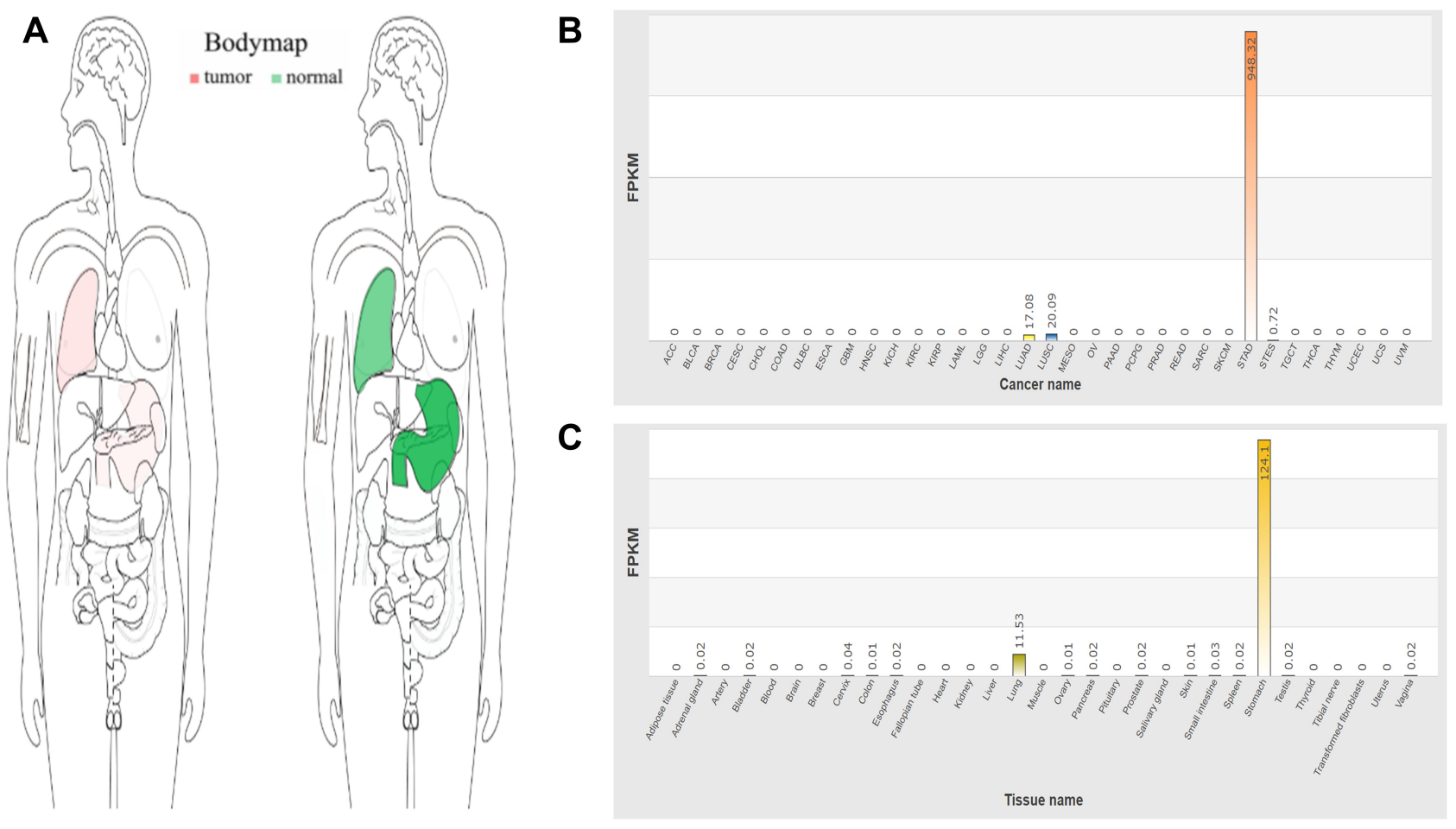

Figure I The expression of GKN2 in human tumors and normal tissues. (A) the median expression of GKN2 in tumor (Red colour) and normal (Green colour) samples in the interactive body map. The expression of GKN2 in different human tumors from TCGA (B) and normal tissues from GTEx (C). 
higher expression was found in breast and esophageal cancers (Figure 2A). And among this, most top dataset were found in gastric cancer ( 9 analyses) and lung cancer (9 analyses) (Supplementary Table 1). Next, we further examined GKN2 expression in various cancers with RNA-sequencing expression data from the TCGA and the GTEx projects, the expression profile of GKN2 across all tumor samples and paired normal tissues was displayed in Figure 2B. There were significantly different in GKN2 expression between LUAD, LUSC, STAD, and their paired normal tissues, however, GKN2 was expressed at similar levels in other tumors and the corresponding normal samples (Figure 2B).

Taken together, these data suggested that GKN2 might play a significant role in lung and gastric cancers, and thus the subsequent studies would mainly focus on the unique role of GKN2 in these two kinds of human cancers.

\section{Genetic Alteration, mRNA Expression, Methylation Level of GKN2 in Lung and Gastric Cancers}

The genetic alterations of GKN2 in lung and gastric cancers were analyzed with cBioPortal. As a result, GKN2 was altered in 3\%, 5\%, 4\% of the included LUAD, LUSC, STAD samples, respectively (Figure 3A).

The mRNA expression and the methylation level of GKN2 in lung and gastric cancers were analyzed with UALCAN. As shown in Figure 3B-C, GKN2 was significantly down-regulated in tumor samples of LUAD, LUSC, and STAD when compared with the corresponding normal samples (Figure 3B). And significant hyper-methylation of GKN2 was found in LUAD and LUSC tumor samples (Figure 3C).

We also assessed the correlation between GKN2 expression and the pathological stage. As displayed in Supplementary Figure 2, a significant correlation was found between the GKN2 expression and LUSC pathological stage $(\mathrm{P}<0.05)$.

\section{Prognostic Value of GKN2 Expression in Lung and Gastric Cancers}

To explore the correlation between GKN2 mRNA expression and prognosis in lung and gastric cancers, we investigated the effects of GKN2 expression on survival via Kaplan-Meier plotter. All in all, low mRNA expression of GKN2 was significantly associated
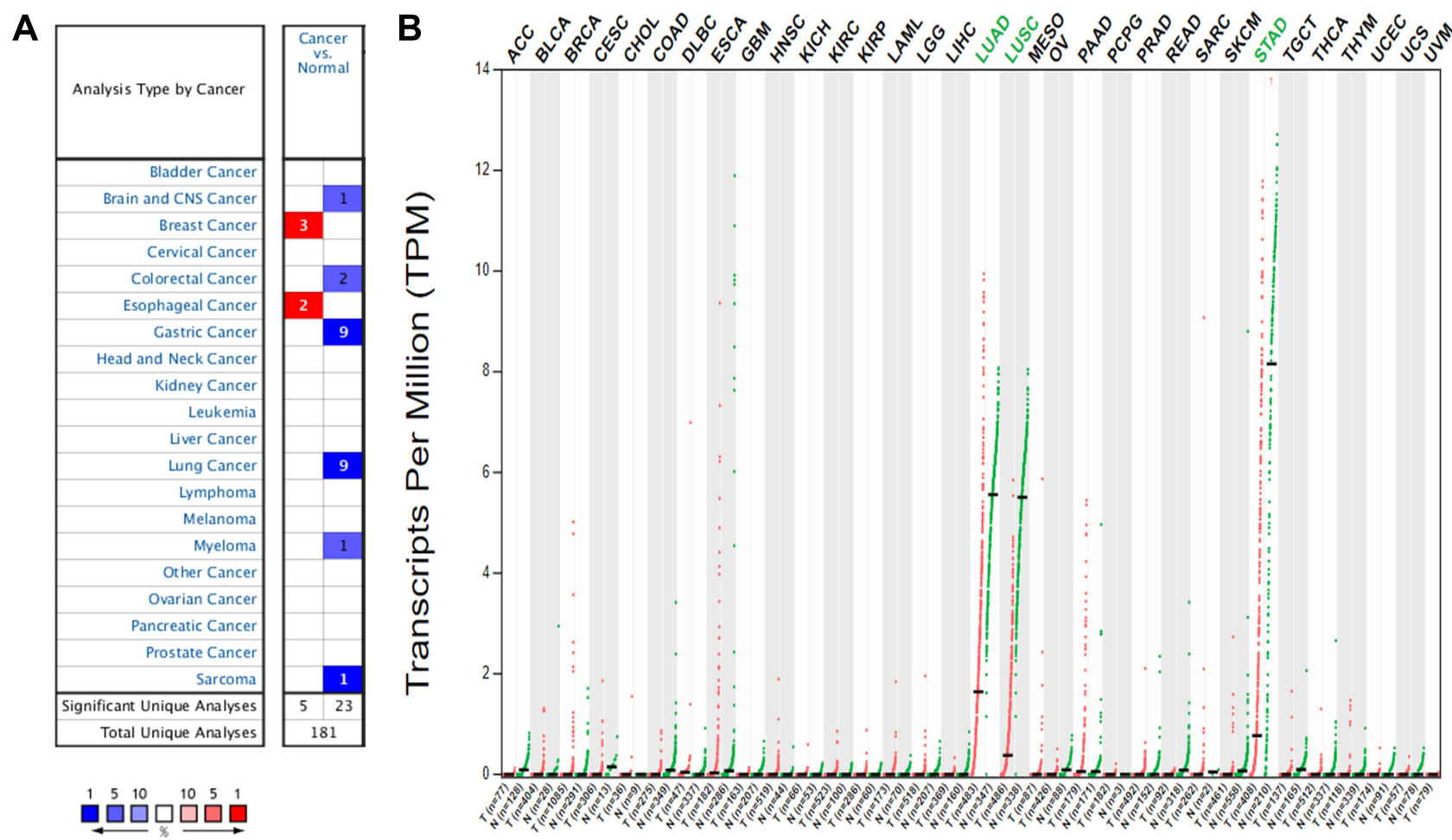

Figure 2 The gene expression level of GKN2 in human tumors compared to paired normal samples. (A) Differential expression of GKN2 in the Oncomine database. (B) The expression profile of GKN2 across all tumor samples and paired normal tissues in the GEPIA database. 
A

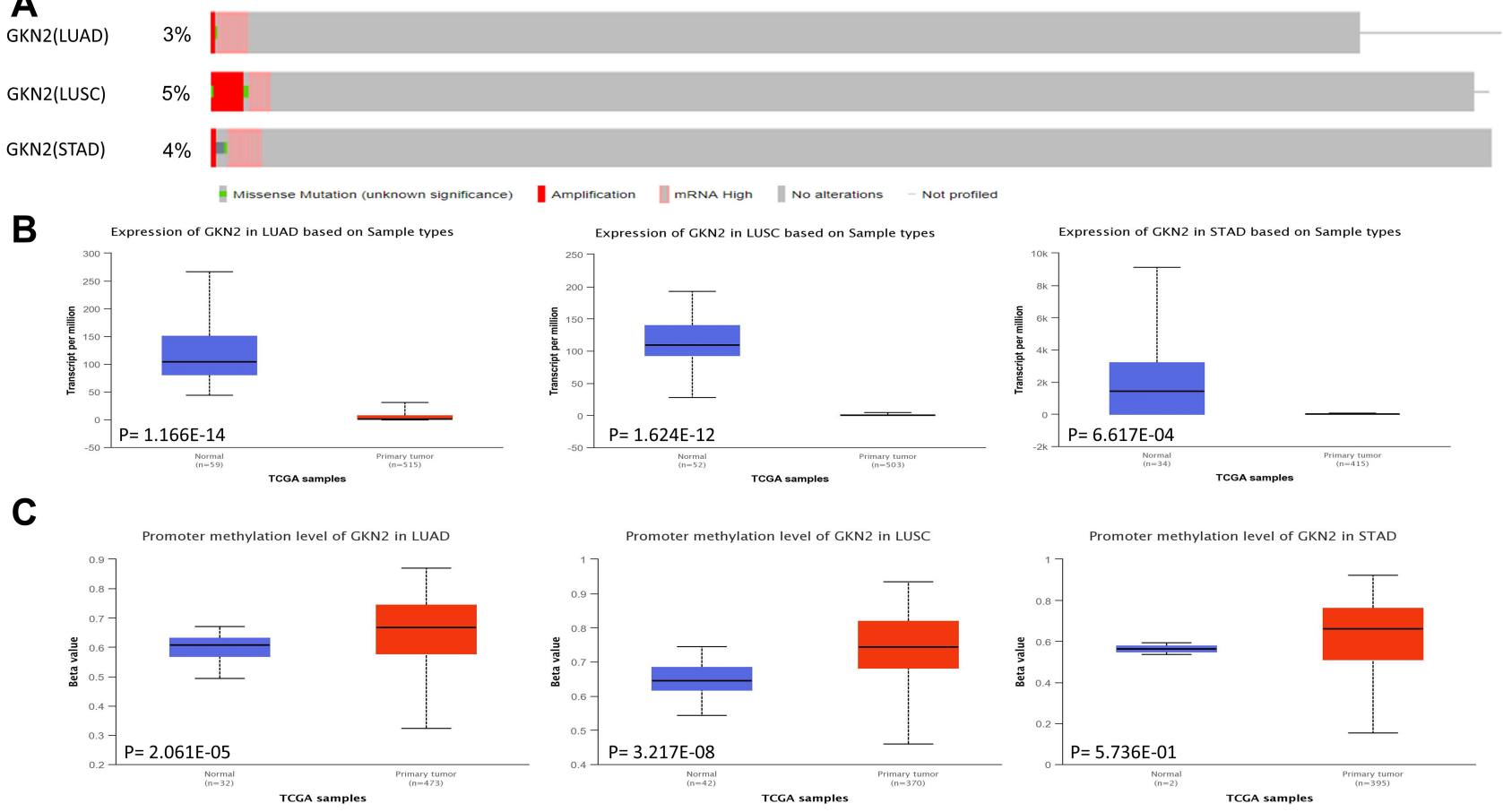

Figure 3 Genetic alteration (A), mRNA expression (B), methylation level (C) of GKN2 in lung and gastric cancer.

with worse OS (HR $=0.83,95 \%$ CI: $0.71-0.98, \mathrm{P}=0.03) \quad 95 \% \mathrm{CI}:(0.58-0.94, \mathrm{P}=0.013)$ and worse PPS $(\mathrm{HR}=$ in lung cancer (Figure 4). And GKN2 low expression 0.68, 95\% CI: $0.52-0.9, \mathrm{P}=0.006$ ) in gastric cancer was found to be correlated with inferior FP (HR $=0.74$, (Figure 4).
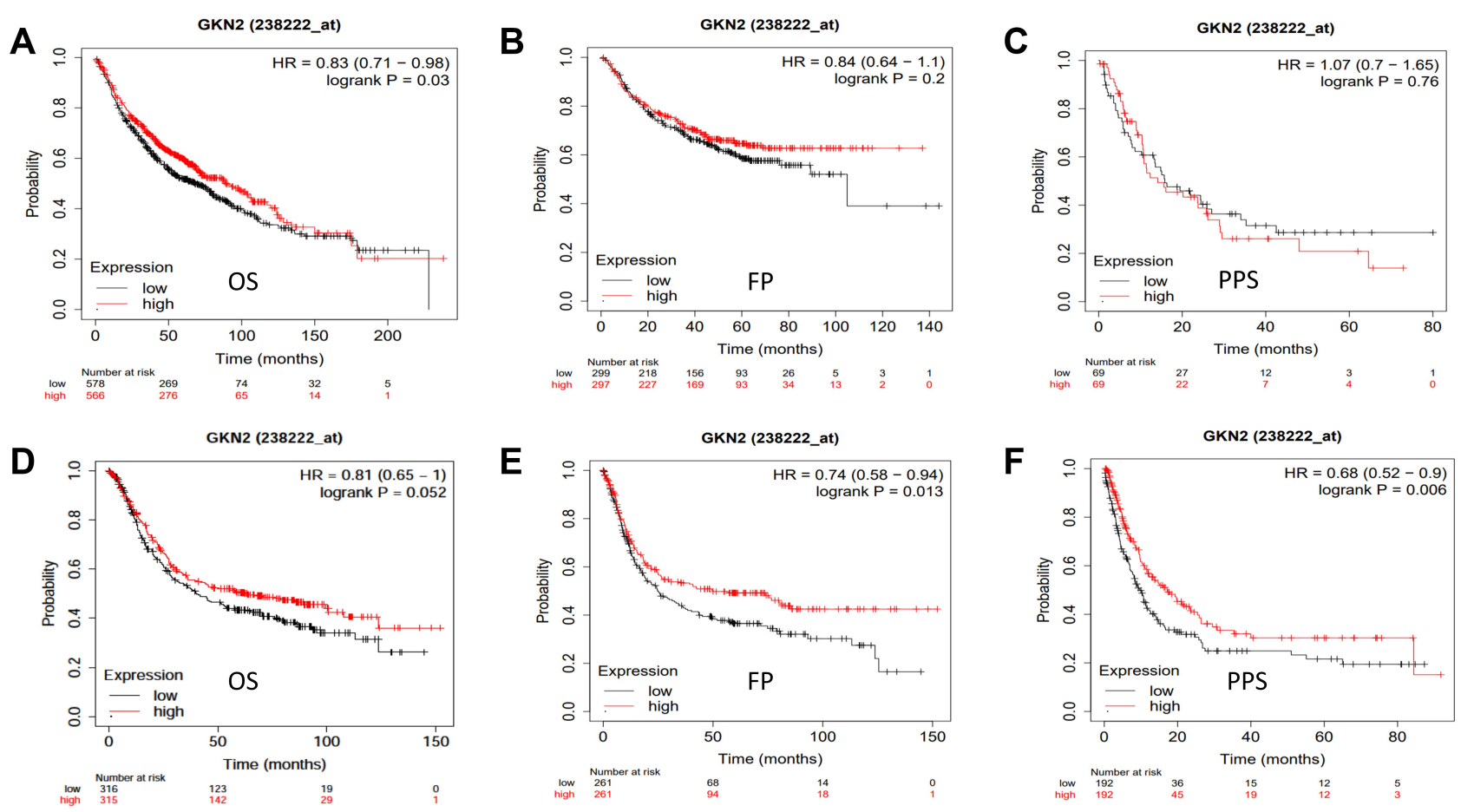

Figure 4 Survival curves of low expression of GKN2 in lung cancer (A-C) and gastric cancer (D-F) from Kaplan-Meier plotter. 


\section{The Relationship Between GKN2} Expression Levels and Clinical Variables in Lung and Gastric Cancers

And then, we further studied the relationship between GKN2 mRNA expression levels and clinical variables in lung and gastric cancer patients with the Kaplan-Meier plotter. The results of prognostic values of GKN2 in clinicopathological subtypes regarding OS, FP, PPS in lung, and gastric cancer were displayed in Supplementary Table 2 and Supplementary Table 3, respectively.

For lung cancer, low GKN2 mRNA expression was correlated with worse OS in male patients, and inferior FP in patients with never smoked history, lung adenocarcinoma, and treatments (only surgical margins negative, or without chemotherapy) (Supplementary Table 2).

For gastric cancer, compared with high levels of GKN2, low GKN2 transcriptional levels were correlated with worse OS/FP/PPS in male patients and cases in stage 3 disease, stage T3, or stage M0 status (Supplementary Table 3). Moreover, GKN2 expression had a significant prognostic correlation with the $\mathrm{N}$ stage, low GKN2 mRNA expression was significantly related with worse OS/FP/PPS in patients with lymph node metastasis (Supplementary Table 3). Decreased GKN2 expression was also significantly related to worse OS/FP in patients with poorly differentiated differentiation, lower GKN2 was correlated with worse prognosis after stratification by Lauren classification (diffuse and mixed for shorter OS, mixed for inferior FP, diffuse for worse PPS) (Supplementary Table 3).

\section{Prognostic Value of DNA Methylation of GKN2 in LUAD, LUSC, and STAD}

DNA methylation has been recognized as an important biological process of tumorigenesis and cancer development. We investigated the effects of DNA methylation of GKN2 on the survival of LUAD, LUSC, and STAD cases via the MethSurv platform.

The heat map of DNA methylation results of GKN2 in LUAD, LUSC and STAD were displayed in Figure 5A-C. And the effect of the DNA methylation patterns of GKN2 in LUAD, LUSC, and STAD was also identified (Figure 6A-H). Among them, the significant prognostic values were observed in TSS1500-Open_Sea-cg02298327 ( $\mathrm{P}=0.0097)$, TSS1500-Open_Sea-cg24550525 ( $\mathrm{P}=0.018)$, Body-Open _Sea-cg18543306 $(\mathrm{P}=0.047)$ in LUAD, and TSS1500-Open _Sea-cg02298327 $(\mathrm{P}=0.016)$ in LUSC.

\section{Immune Cell Infiltration of GKN2 in} Patients with LUAD, LUSC, and STAD

To explore the potential mechanism by which GKN2 expression affects clinical outcome, we studied the correlation between GKN2 and tumor-infiltrating immune cells with TIMER. Among the six tumor-infiltrating immune cells, GKN2 expression was found only to be positively correlated with the infiltration of macrophages $(\mathrm{P}<0.05)$ in LUAD. And for LUSC, GKN2 expression positively correlated with all six immune infiltrates (B cells, CD4+ $\mathrm{T}$ cells, CD8 $+\mathrm{T}$ cells, Neutrophils, Macrophages, and Dendritic cells) (all $\mathrm{P}<0.05$ ). For STAD, there were significantly positive correlations between GKN2 expression and the infiltration of immune cells (B cells, and CD8+ $\mathrm{T}$ cells) $(\mathrm{P}<0.05)$ (Figure 7). In addition, we also explored the correlations between somatic copy number alterations (CAN) of GKN2 and abundance of immune infiltrates in LUAD, LUSC, and STAD, these results were displayed in Supplementary Figure 3. Furthermore, the cox proportional hazard model of GKN2 and six tumor-infiltrating immune cells in LUAD, LUSC, STAD was performed. As shown in Supplementary Table 4. B_cellandCD4_Tcell, GKN2, and Macrophage were found to be significantly related to the clinical outcome of LUAD, LUSC, and STAD patients, respectively.

\section{Correlation Analysis Between GKN2 Expression and Neutrophils and Macrophage Polarization Markers in LUAD, LUSC and STAD}

Since neutrophils and macrophages played vital roles in immunity and tumor development, we analyzed the correlation coefficients between GKN2 expression and specific markers of neutrophils and macrophage polarization (monocytes, TAMs, M1, and M2 macrophages) in LUAD, LUSC, and STAD.

MPO, PTPRC, and CD15 were applied as neutrophils markers (Supplementary Figure 4), the results from scatterplots displayed that there were significant correlations between GKN2 and neutrophil markers (MPO and PTPRC) in LUSC, and neutrophil markers (PTPRC) in STAD.

CD14, CD36, and ITGAM were identified as markers for monocytes, CD68, CCL2, and CSF1R for TAMs (tumor-associated macrophages), NOS2, CXCL10, and TNF for M1 macrophages, and CCL18, CD163, and 

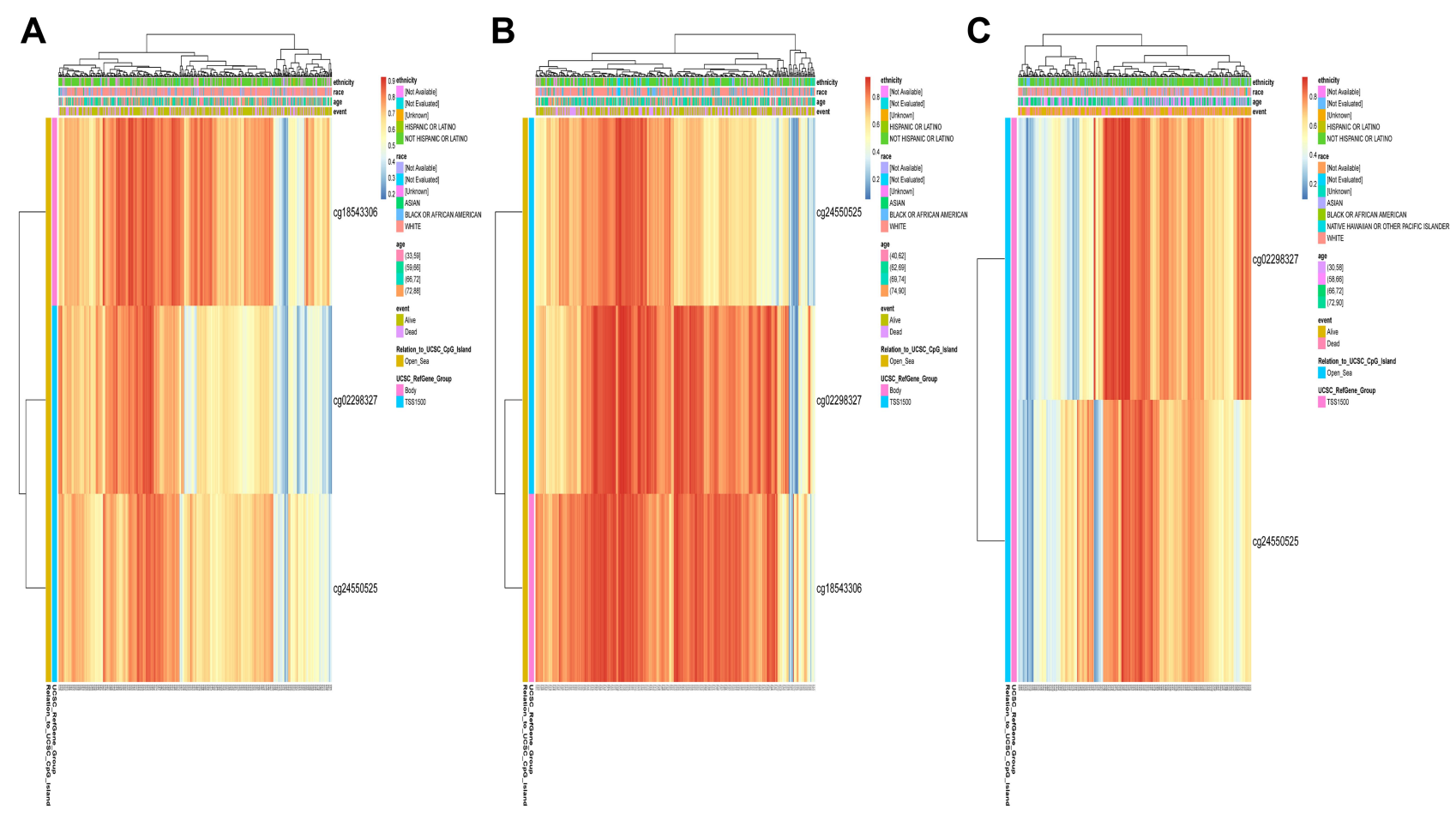

Figure 5 The heat map of DNA methylation of GKN2 in LUAD (A), LUSC (B), STAD (C).
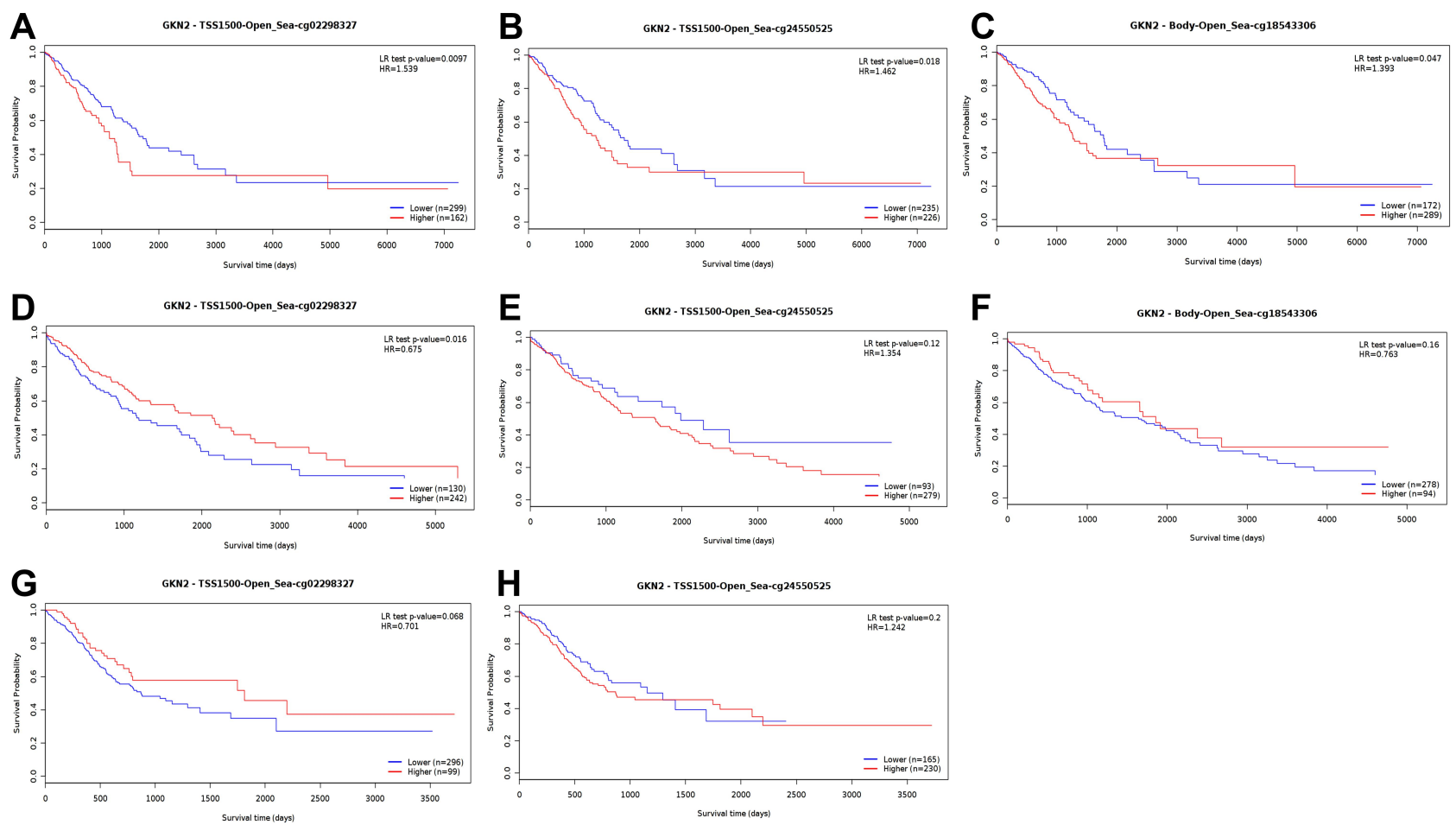

Figure 6 The prognostic value of DNA methylation of GKN2 in LUAD (A-C), LUSC (D-F) and STAD (G, H).

IL10 for M2 macrophages. In LUAD (Figure 8), the results from scatterplots showed that there were significant correlations between GKN2 and the immune markers of monocytes (CD36), M1 (NOS2, CXCL10), and M2 macrophages (CCL18, IL10) in tumor tissues. In LUSC (Figure 9), there were significant correlations 


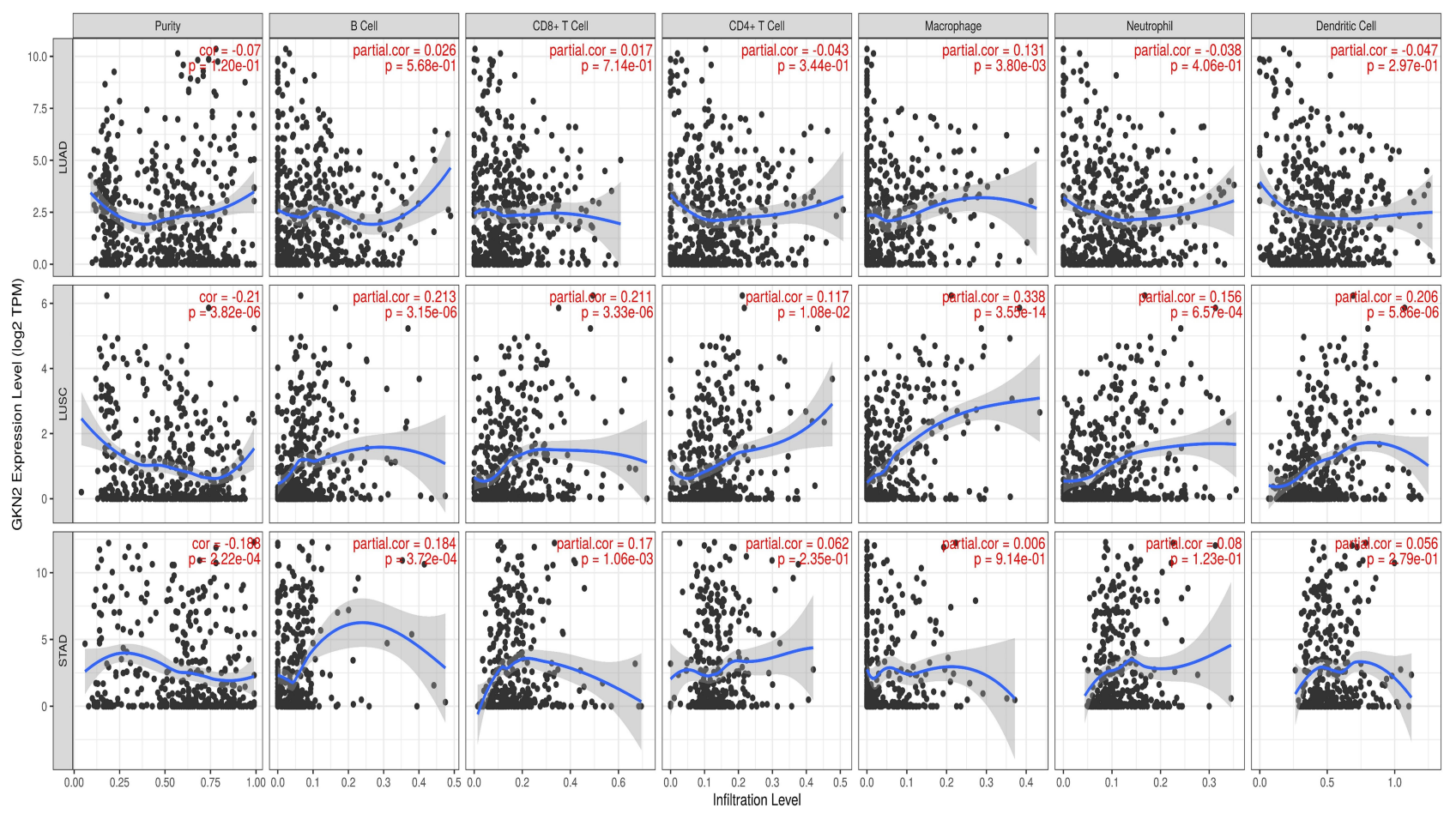

Figure 7 The correlation between GKN2 and immune cell infiltration in LUAD, LUSC, STAD (TIMER).

between GKN2 and most immune markers of monocytes, between GKN2 and immune markers of monocytes TAMs, M1, and M2 macrophages in tumor tissues. In (CD36, ITGAM) and M1 macrophages (TNF) in tumor STAD (Figure 10), significant correlations were found tissues.
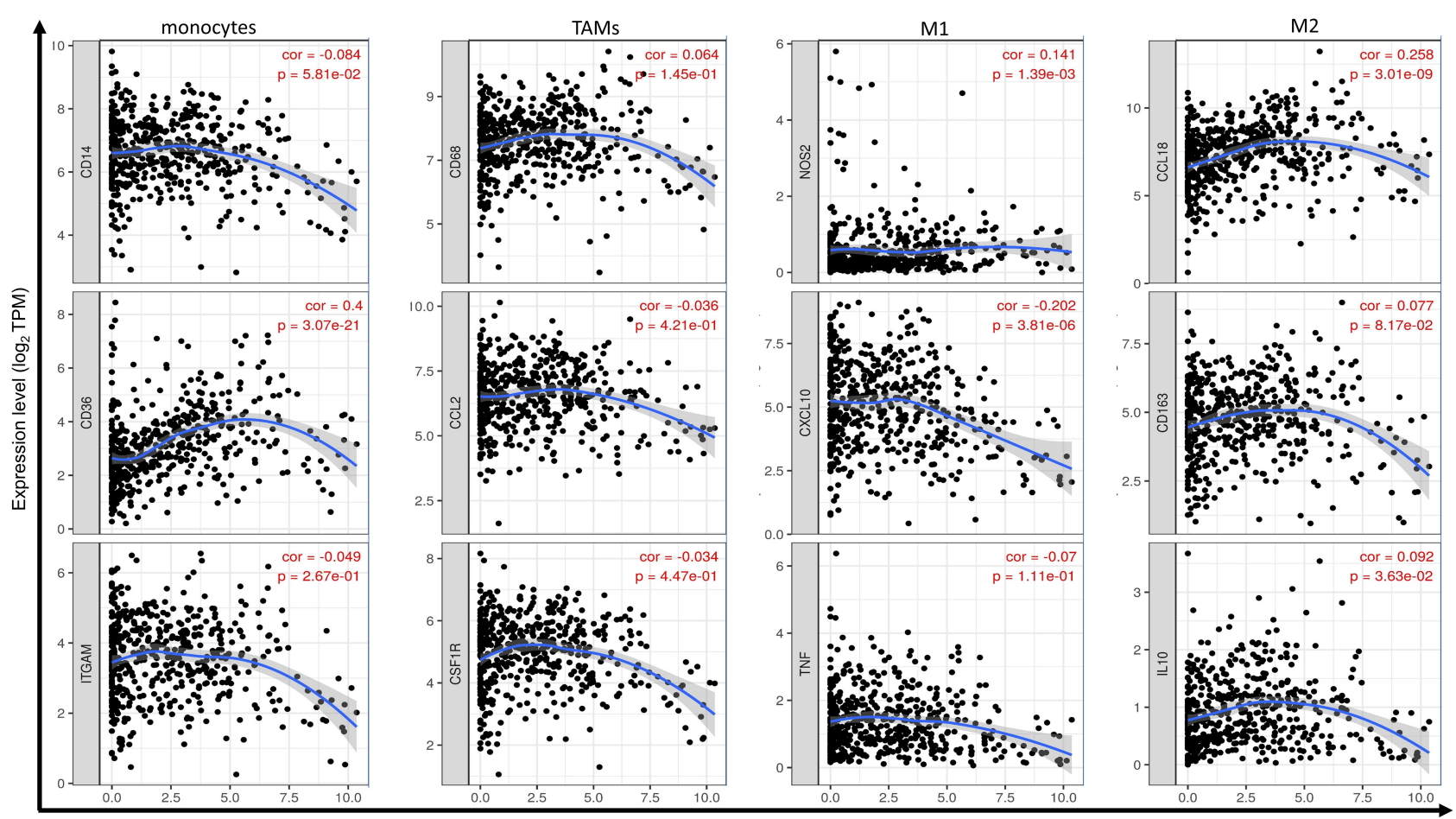

GKN2 expression level $\left(\log _{2}\right.$ TPM)

Figure 8 Scatterplots of correlation between GKN2 expression and the expression of gene markers of macrophage polarization in LUAD $(n=5 \mid 5)$. 
In addition, the relationships between GKN2 and the above gene markers in cancers were assessed using the GEPIA database, the results were generally consistent with those of the TIMER (Supplementary Table 5). The results implied that GKN2 might play an important role in the regulation of macrophage polarization in LUAD, LUSC, and STAD.

\section{Discussion}

Gastrokine (GKN) family comprises three secreted proteins, and GKN2 is an important member of this family. ${ }^{35} \mathrm{GKN} 2$ is a cytoplasmic protein that mainly localizes to the epithelial cells of the normal human gastric mucosa. GKN2 was highly expressed in normal gastric mucosa but was decreased or even absent in STAD tissues, especially in the diffuse subtype. ${ }^{4}$ Trefoil factor family (TFF) peptides are a group of small peptides with the characteristic of three-loop structure named the trefoil domain. These peptides have important functionalities, including interactions with the immune system, involvement in mucosal protection and repair, as well as in tumorigenesis. ${ }^{36-38}$ Certain interactions at the structure and function levels between the GKN and TFF families were also identified. Previous reports showed that GKN2 could bind with TFF $2,{ }^{39}$ as well as TFF1 at a protein level. ${ }^{40,41}$ Moreover, the disruption of co-expression between GKN2 and TFF1 was found to be related to a more invasive and metastatic phenotype induced by TFF $1 .{ }^{40,42}$ GKN2 also showed effects on the behaviors of tumor cells, GKN2 absence could promote proliferation, migration, and metastasis by causing the degradation of extracellular matrix components. ${ }^{14}$ Notably, GKN2 was involved in immune and inflammation response of the tumor microenvironment, GKN2 shown an anti-inflammatory role in gastric tumor progression, ${ }^{18,43}$ and elevated GKN2 was associated with a more favorable outcome in multiple cancers. ${ }^{6,12,13}$ Therefore, our study aimed to provide evidence that GKN2 could serve as a prognostic marker as well as to shed light on the potential effects of GKN2 in the tumor microenvironment.

In this study, GKN2 transcription level profiles in various kinds of normal tissues and tumors were analyzed with datasets from GEPIA, SEGreg, and Oncomine. In the comparison of various normal tissues, we observed GKN2 expression was much higher in the stomach and followed by lung tissue. And among different kinds of tumors, the GKN2 mRNA expression level was obviously higher in STAD and followed by LUAD and LUSC. According to the analysis results of the Oncomine, the most top datasets that GKN2 differentially expressed were gastric and lung cancer, and the data from TCGA and the GTEx projects also confirmed that GKN2 expression level was
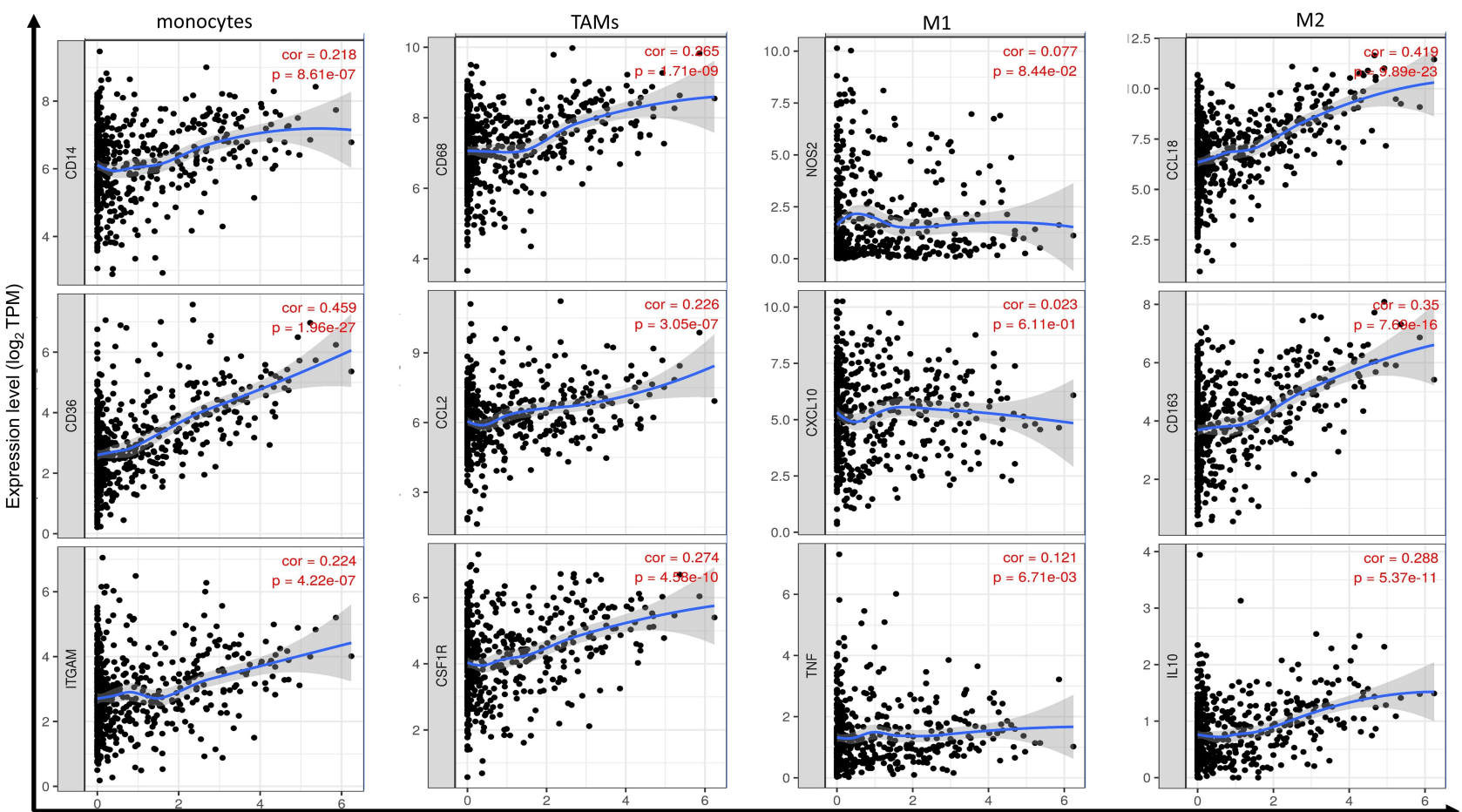

GKN2 expression level $\left(\log _{2}\right.$ TPM)

Figure 9 Scatterplots of correlation between GKN2 expression and the expression of gene markers of macrophage polarization in LUSC $(n=501)$. 


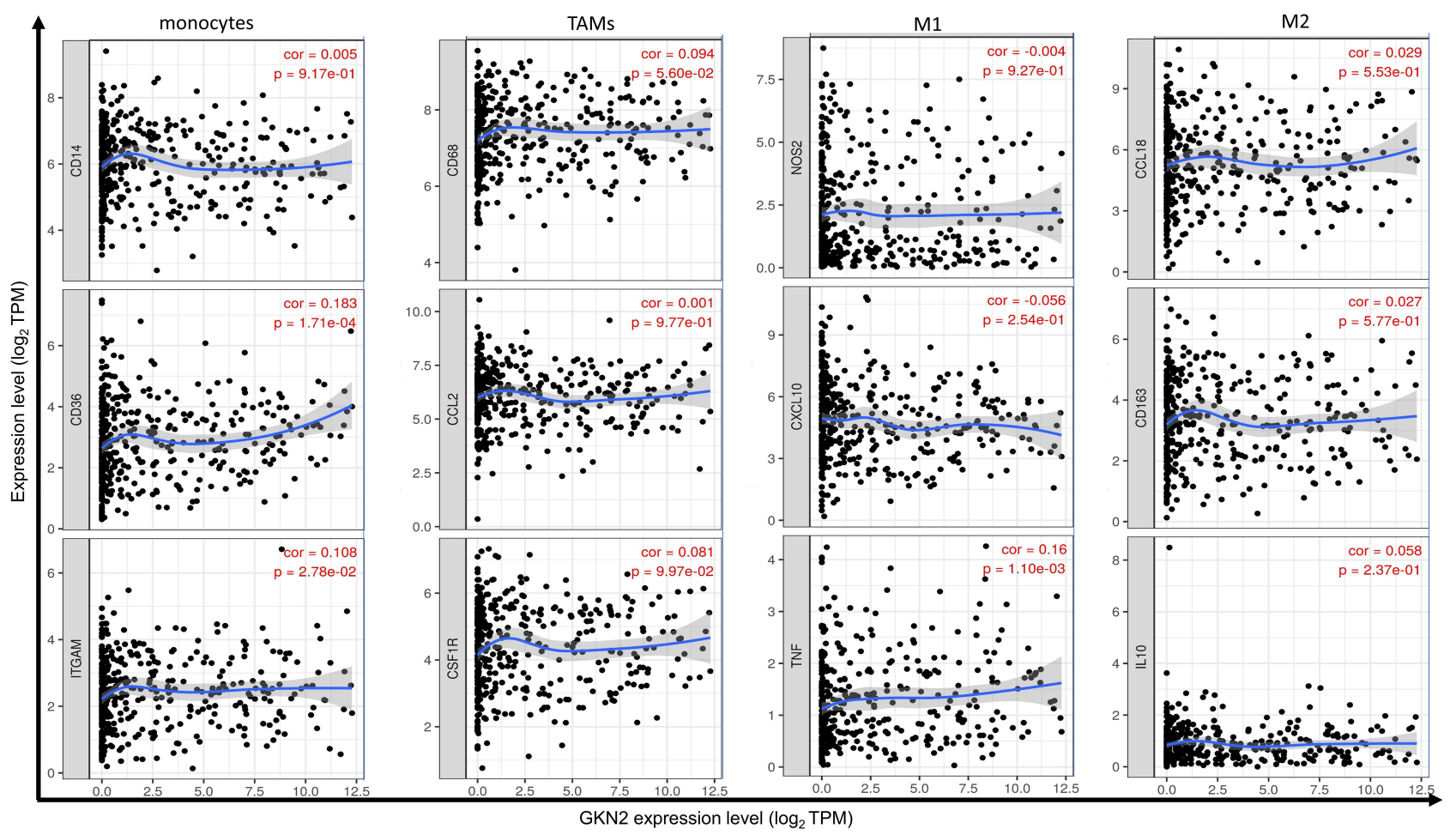

Figure 10 Scatterplots of correlation between GKN2 expression and the expression of gene markers of macrophage polarization in STAD $(n=4 I 5)$.

significantly lower in tumor samples in LUAD, LUSC, and STAD when compared with their paired normal tissues. These indicated the unique and important roles of GKN2 in gastric and lung cancers.

According to the cBioPortal, the GKN2 genetic alterations were found in $3 \%, 5 \%, 4 \%$ of the LUAD, LUSC, STAD samples, respectively. The results from UALCAN further shown the significantly down-regulated expression level of GKN2 in LUAD, LUSC, and STAD. And the hyper-methylation phenomenon of GKN2 was observed in LUAD and LUSC tumor samples. In addition, there was a significant correlation between GKN2 expression and LUSC pathological stage. Furthermore, the clinical values of GKN2 expression were also explored in lung and gastric cancer using the Kaplan-Meier plotter. Low GKN2 mRNA expression indicated worse OS in patients with lung cancer and inferior FP/PPS in cases with gastric cancer. And compared with higher expression of GKN2, decreased expression of GKN2 was revealed to be associated with worse OS/FP/PPS for lung cancer patients in stage 3 disease, stage T3, or stage M0 status. And in gastric cancer, the low GKN2 expression level was significantly related with worse OS/FP/PPS in patients with lymph node metastasis, decreased GKN2 expression was also significantly related with worse OS/FP in patients with poorly differentiated differentiation, lower GKN2 was correlated with worse prognosis after stratification by Lauren classification. In summary, these results provided strong evidence that GKN2 is a powerful tumor marker to predict clinical outcomes in the lung and gastric cancers.

We found that GKN2 expression was related to the infiltration of tumor immune cells in the lung and gastric cancers. GKN2 expression was found to be positively correlated with macrophages infiltration in LUAD, and six immune infiltrates, including B cells, CD4 $+\mathrm{T}$ cells, CD8 $+\mathrm{T}$ cells, Neutrophils, Macrophages, and Dendritic cells in LUSC, and B cells and CD8+ T cells in STAD. And the correlations between GKN2 CAN and immune infiltrates abundance in LUAD, LUSC, and STAD were assessed, and B_cellandCD4_Tcell, GKN2, Macrophage were found to be significantly related to the clinical outcome of LUAD, LUSC and STAD patients, respectively. Interestingly, we also performed correlation analysis between GKN2 expression and neutrophils and macrophage polarization markers in LUAD, LUSC and STAD. We found significant correlations between GKN2 and neutrophil markers (MPO and PTPRC) in LUSC, and neutrophil marker (PTPRC) in STAD, significant correlations between GKN2 and the immune markers of monocytes 
(CD36), M1 (NOS2, CXCL10), and M2 macrophages (CCL18, IL10) were found in LUAD, significant correlations were revealed between GKN2 and most immune markers of monocytes, TAMs, M1, and M2 macrophages in tumor tissues of LUSC, and significant correlations were found between GKN2 and immune markers of monocytes (CD36, ITGAM) and M1 macrophages (TNF) in tumor tissues of STAD. These correlations indicated a potential mechanism that GKN2 regulated the neutrophils and macrophage in LUSC, LUAD, and STAD. Therefore, GKN2 might play an important role in immune cell recruitment and functional regulation in the tumor microenvironment.

Some limitations of this study should be acknowledged. This work was based on data from multiple public databases, the biases resulting from confounding factors might exist. And the exact mechanisms of GKN2 in the regulation of the infiltrating of tumor immune cells are largely uncovered and needed to be explored in future researches.

In conclusion, this work provided comprehensive evidence of GKN2 as a valuable clinical biomarker and immunotherapy target in the lung and gastric cancers. GKN2 transcription level was significantly related to survival outcomes and tumor-infiltrating immune cells in lung and gastric cancer. GKN2 might play an important role in the infiltrating of tumor immune cells and can be applied as a prognostic biomarker in lung and gastric cancer.

\section{Data Sharing Statement}

All the figures and tables adopted to support the findings of the present study are included in the article.

\section{Acknowledgments}

We would like to forward our gratitude to the anonymous reviewers and editors for their careful work and valuable comments.

\section{Disclosure}

The authors declare that they have no conflicts of interest.

\section{References}

1. Du JJ, Dou KF, Peng SY, et al. [Down-regulated full-length novel gene GDDR and its effect on gastric cancer]. Zhonghua Yi Xue Za Zhi. 2003;83(13):1166-1168. Chinese.

2. Westley BR, Griffin SM, May FE. Interaction between TFF1, a gastric tumor suppressor trefoil protein, and TFIZ1, a brichos domain-containing protein with homology to SP-C. Biochemistry. 2005;44(22):7967-7975. doi:10.1021/bi047287n
3. Fagerberg L, Hallström BM, Oksvold P, et al. Analysis of the human tissue-specific expression by genome-wide integration of transcriptomics and antibody-based proteomics. Mol Cell Proteomics. 2014;13 (2):397-406. doi:10.1074/mcp.M113.035600

4. Moss SF, Lee JW, Sabo E, et al. Decreased expression of gastrokine 1 and the trefoil factor interacting protein TFIZ1/GKN2 in gastric cancer: influence of tumor histology and relationship to prognosis. Clin Cancer Res. 2008;14(13):4161-4167. doi:10.1158/1078-0432.Ccr-07-4381

5. Garber ME, Troyanskaya OG, Schluens K, et al. Diversity of gene expression in adenocarcinoma of the lung. Proc Natl Acad Sci U S A. 2001;98(24):13784-13789. doi:10.1073/pnas.241500798

6. Smith JJ, Deane NG, Wu F, et al. Experimentally derived metastasis gene expression profile predicts recurrence and death in patients with colon cancer. Gastroenterology. 2010;138(3):958-968. doi:10.1053/j. gastro.2009.11.005

7. Liang Y, Diehn M, Watson N, et al. Gene expression profiling reveals molecularly and clinically distinct subtypes of glioblastoma multiforme. Proc Natl Acad Sci U S A. 2005;102(16):5814-5819. doi:10.1073/pnas.0402870102

8. Kim SM, Park YY, Park ES, et al. Prognostic biomarkers for esophageal adenocarcinoma identified by analysis of tumor transcriptome. PLoS One. 2010;5(11):e15074. doi:10.1371/journal.pone.0015074

9. Hao Y, Triadafilopoulos G, Sahbaie P, Young HS, Omary MB, Lowe AW. Gene expression profiling reveals stromal genes expressed in common between Barrett's esophagus and adenocarcinoma. Gastroenterology. 2006;131(3):925-933. doi:10.1053/j.gastro.2006.04.026

10. Finak G, Bertos N, Pepin F, et al. Stromal gene expression predicts clinical outcome in breast cancer. Nat Med. 2008;14(5):518-527. doi:10.1038/nm1764

11. Turashvili G, Bouchal J, Baumforth K, et al. Novel markers for differentiation of lobular and ductal invasive breast carcinomas by laser microdissection and microarray analysis. BMC Cancer. 2007;7 (1):55. doi:10.1186/1471-2407-7-55

12. Okayama H, Kohno T, Ishii Y, et al. Identification of genes upregulated in ALK-positive and EGFR/KRAS/ALK-negative lung adenocarcinomas. Cancer Res. 2012;72(1):100-111. doi:10.1158/ 0008-5472.Can-11-1403

13. Ivshina AV, George J, Senko O, et al. Genetic reclassification of histologic grade delineates new clinical subtypes of breast cancer. Cancer Res. 2006;66(21):10292-10301. doi:10.1158/0008-5472.Can-05-4414

14. Dai J, Zhang N, Wang J, Chen M, Chen J. Gastrokine-2 is downregulated in gastric cancer and its restoration suppresses gastric tumorigenesis and cancer metastasis. Tumour Biol. 2014;35 (5):4199-4207. doi:10.1007/s13277-013-1550-0

15. Baus-Loncar M, Lubka M, Pusch CM, Otto WR, Poulsom R, Blin N. Cytokine regulation of the trefoil factor family binding protein GKN2 (GDDR/TFIZ1/blottin) in human gastrointestinal epithelial cells. Cell Physiol Biochem. 2007;20(1-4):193-204. doi:10.1159/000104166

16. Sharman MJ, Bacci B, Santos L, Mansfield CS. Gastrokine mRNA expression in gastric tissue from dogs with helicobacter colonisation but without inflammatory change during treatment. Vet Immunol Immunopathol. 2017;187:28-34. doi:10.1016/j.vetimm.2017.03.004

17. Chung Nien Chin S, O'Connor L, Scurr M, et al. Coordinate expression loss of GKN1 and GKN2 in gastric cancer via impairment of a glucocorticoid-responsive enhancer. Am J Physiol Gastrointest Liver Physiol. 2020;319(2):G175-g188. doi:10.1152/ajpgi.00019.2020

18. Zhang Z, Xue H, Dong Y, et al. Inhibition of GKN2 attenuates acute gastric lesions through the NLRP3 inflammasome. Advan Wound Care. 2020;9(5):219-232. doi:10.1089/wound.2019.0957

19. Kim O, Yoon JH, Choi WS, et al. GKN2 contributes to the homeostasis of gastric mucosa by inhibiting GKN1 activity. J Cell Physiol. 2014;229(6):762-771. doi:10.1002/jcp.24496

20. Tang Z, Li C, Kang B, Gao G, Li C, Zhang Z. GEPIA: a web server for cancer and normal gene expression profiling and interactive analyses. Nucleic Acids Res. 2017;45(W1):W98-w102. doi:10.1093/ nar/gkx247 
21. Tang Q, Zhang Q, Lv Y, Miao YR, Guo AY. SEGreg: a database for human specifically expressed genes and their regulations in cancer and normal tissue. Brief Bioinform. 2019;20(4):1322-1328. doi:10.1093/bib/bbx173

22. Rhodes DR, Yu J, Shanker K, et al. ONCOMINE: a cancer microarray database and integrated data-mining platform. Neoplasia (New York, NY). 2004;6(1):1-6. doi:10.1016/s1476-5586(04)80047-2

23. Chandrashekar DS, Bashel B, Balasubramanya SAH, et al. UALCAN: a portal for facilitating tumor subgroup gene expression and survival analyses. Neoplasia (New York, NY). 2017;19 (8):649-658. doi:10.1016/j.neo.2017.05.002

24. Cerami E, Gao J, Dogrusoz U, et al. The cBio cancer genomics portal: an open platform for exploring multidimensional cancer genomics data. Cancer Discov. 2012;2(5):401-404. doi:10.1158/21598290.Cd-12-0095

25. Gao J, Aksoy BA, Dogrusoz U, et al. Integrative analysis of complex cancer genomics and clinical profiles using the cBioPortal. Sci Signal. 2013;6(269):pl1. doi:10.1126/scisignal.2004088

26. Nagy Á, Lánczky A, Menyhárt O, Györffy B. Validation of miRNA prognostic power in hepatocellular carcinoma using expression data of independent datasets. Sci Rep. 2018;8(1):9227. doi:10.1038/ s41598-018-27521-y

27. Modhukur V, Iljasenko T, Metsalu T, Lokk K, Laisk-Podar T, Vilo J. MethSurv: a web tool to perform multivariable survival analysis using DNA methylation data. Epigenomics. 2018;10(3):277-288. doi:10.2217/epi-2017-0118

28. Li B, Severson E, Pignon JC, et al. Comprehensive analyses of tumor immunity: implications for cancer immunotherapy. Genome Biol. 2016;17(1):174. doi:10.1186/s13059-016-1028-7

29. Li T, Fan J, Wang B, et al. TIMER: a web server for comprehensive analysis of tumor-infiltrating immune cells. Cancer Res. 2017;77(21): e108-e110. doi:10.1158/0008-5472.Can-17-0307

30. Auffray C, Sieweke MH, Geissmann F. Blood monocytes: development, heterogeneity, and relationship with dendritic cells. Annu Rev Immunol. 2009;27:669-692. doi:10.1146/annurev.immunol.021908. 132557

31. Geissmann F, Manz MG, Jung S, Sieweke MH, Merad M, Ley K. Development of monocytes, macrophages, and dendritic cells. Science (New York, NY). 2010;327(5966):656-661. doi:10.1126/ science. 1178331
32. Quatromoni JG, Eruslanov E. Tumor-associated macrophages: function, phenotype, and link to prognosis in human lung cancer. $\mathrm{Am}$ J Transl Res. 2012;4(4):376-389.

33. Geissmann F, Jung S, Littman DR. Blood monocytes consist of two principal subsets with distinct migratory properties. Immunity. 2003;19(1):71-82. doi:10.1016/s1074-7613(03)00174-2

34. Zhang X, Lan Y, Xu J, et al. CellMarker: a manually curated resource of cell markers in human and mouse. Nucleic Acids Res. 2019;47 (D1):D721-D728. doi:10.1093/nar/gky900

35. Menheniott TR, Kurklu B, Giraud AS. Gastrokines: stomach-specific proteins with putative homeostatic and tumor suppressor roles. $A m$ $J$ Physiol Gastrointest Liver Physiol. 2013;304(2):G109-21. doi:10.1152/ajpgi.00374.2012

36. Katoh M. Trefoil factors and human gastric cancer (review). Int J Mol Med. 2003;12(1):3-9.

37. Kjellev S. The trefoil factor family - small peptides with multiple functionalities. Cell Mol Life Sci. 2009;66(8):1350-1369. doi:10. 1007/s00018-008-8646-5

38. Matsubara D, Yoshimoto T, Soda M, et al. Reciprocal expression of trefoil factor-1 and thyroid transcription factor-1 in lung adenocarcinomas. Cancer Sci. 2020;111(6):2183-2195. doi:10.1111/ cas. 14403

39. Otto WR, Patel K, McKinnell I, et al. Identification of blotting: a novel gastric trefoil factor family-2 binding protein. Proteomics. 2006;6(15):4235-4245. doi:10.1002/pmic.200500911

40. May FE, Griffin SM, Westley BR. The trefoil factor interacting protein TFIZ1 binds the trefoil protein TFF1 preferentially in normal gastric mucosal cells but the co-expression of these proteins is deregulated in gastric cancer. Int J Biochem Cell Biol. 2009;41 (3):632-640. doi:10.1016/j.biocel.2008.07.015

41. Kouznetsova I, Laubinger W, Kalbacher H, et al. Biosynthesis of gastrokine-2 in the human gastric mucosa: restricted spatial expression along the antral gland axis and differential interaction with TFF1, TFF2 and mucins. Cell Physiol Biochem. 2007;20 (6):899-908. doi:10.1159/000110450

42. Otto WR, Thim L. Trefoil factor family-interacting proteins. Cell Mol Life Sci. 2005;62(24):2939-2946. doi:10.1007/s00018-005-5482-8

43. Menheniott TR, O'Connor L, Chionh YT, et al. Loss of gastrokine-2 drives premalignant gastric inflammation and tumor progression. J Clin Invest. 2016;126(4):1383-1400. doi:10.1172/jci82655
Journal of Inflammation Research

\section{Publish your work in this journal}

The Journal of Inflammation Research is an international, peerreviewed open-access journal that welcomes laboratory and clinical findings on the molecular basis, cell biology and pharmacology of inflammation including original research, reviews, symposium reports, hypothesis formation and commentaries on: acute/chronic inflammation; mediators of inflammation; cellular processes; molecular mechanisms; pharmacology and novel anti-inflammatory drugs; clinical conditions involving inflammation. The manuscript management system is completely online and includes a very quick and fair peerreview system. Visit http://www.dovepress.com/testimonials.php to read real quotes from published authors. 